\title{
Microbiología de las infecciones causadas por mordeduras de perros y gatos en personas: Una revisión
}

\author{
Microbiology of infections caused by dog and cat bites: A review
}

\author{
Beatriz Pardal-Peláez y Ainhoa Sarmiento-García
}

${ }^{1}$ Departamento de Cirugía. Clínica Odontológica. Facultad de Medicina. Universidad de Salamanca, España.

2Departamento de Construcción y Agronomía. Facultad de Ciencias Ambientales. Universidad de Salamanca, España.

Las autoras declaran no tener conflicto de intereses.

Recibido: 5 de febrero de 2021 / Aceptado: 7 de mayo de 2021

\section{Resumen}

Introducción: Las mordeduras causadas por animales de compañía constituyen el $5 \%$ de las heridas traumáticas registradas en los servicios de urgencias. Objetivo: Conocer los principales agentes infecciosos presentes en las mordeduras provocadas por perros y gatos, tanto de forma individual como conjunta; así como los factores que favorecen la infección. Metodología: Se realizó una búsqueda bibliográfica en PubMed con la siguiente estrategia de búsqueda: ((“Bites, Human”[Mesh]) OR "Bites and Stings"[Mesh]) AND “Infection"'[Mesh]. Se incluyeron 24 trabajos en la síntesis cualitativa escritos en lengua inglesa o española, casos clínicos o descriptivos y publicados entre los años 2000 y 2019. Resultados: Las especies más frecuentemente aisladas fueron Capnocytophaga canimorsus en perros y Pasteurella multocida en gatos. La existencia de comorbilidades en el individuo, la mordedura en la mano, o la presencia de signos de alteración del estado general del individuo mordido fueron considerados como factores de riesgo para el desarrollo de la infección tras la mordedura. Conclusiones: Todos los afectados por mordeduras animales deben recibir asistencia médica y considerar la administración de una pauta de profilaxis antimicrobiana con el fin de reducir el riesgo de shock séptico. Por otro lado, es importante advertir al laboratorio de microbiología de la naturaleza de las muestras clínicas obtenidas para alcanzar el mejor diagnóstico etiológico.

Palabras clave: infección; mascotas; mordeduras; Pasteurella multocida, Capnocytophaga canimorsus.

\section{Abstract}

Background: Bites caused by pets constitute $5 \%$ of the traumatic injuries registered in the emergency services. Aim: To know the main infectious agents present in dog and cat bites, both individually and jointly, in humans, as well as the predisposing factors that favor infection and its spread. Methods: A bibliographic search was carried out in PubMed with the following search strategy: (("Bites, Human" [Mesh]) OR "Bites and Stings" [Mesh]) AND "Infection" [Mesh]. Twenty-four papers were included in the qualitative synthesis written in English or Spanish, clinical or descriptive cases and published between 2000 and 2019. Results: Most frequently isolated species were Capnocytophaga canimorsus in dogs and Pasteurella multocida in cats. The existence of comorbidities in the individual, the bite on the hand, or the presence of signs of alteration of the general state of the bitten individual were considered as risk factors for the development of infection after the bite. Conclusions: All patients with animal bites should receive medical assistance, and the administration of an antibiotic prophylaxis regimen should be considered to reduce the risk of septic shock. Besides, it is important to advise the microbiology laboratory of the nature of the clinical samples obtained in order to reach the best etiological diagnosis.

Keywords: bites; infection; pets; Pasteurella multocida, Capnocytophaga canimorsus.

\footnotetext{
Correspondencia a:

Ainhoa Sarmiento-García

ainhoasarg@gmail.com
} 


\section{- vilumina Infectología al Día}

\section{Introducción}

$\mathrm{E}$ n la última década se han registrado aproximadamente 13 millones de mascotas en España, esto supone que, en torno a $40 \%$ de los hogares españoles tiene al menos una mascota ${ }^{1}$. Este creciente ascenso en el número de animales de compañía justifica el hecho de que las mordeduras causadas por animales constituyan el 5\% de las heridas traumáticas registradas en los servicios de urgencias ${ }^{2-4}$. En las zonas urbanas, la mayoría de las mordeduras son debidas a perros (80-90\%) y gatos $(5-15 \%)^{4-7}$. En $90 \%$ de los casos de mordedura de perro, el animal pertenece a la víctima o a un conocido, y es frecuente que éste fuese intimidado o molestado por la víctima mientras comía. En los gatos se ha descrito que en $98 \%$ de los casos el animal pertenece a la víctima y que la mordedura pudo producirse como consecuencia de una situación estresante para el animal ${ }^{8,9}$.

Por otra parte, ha sido considerado que las mordeduras de perro son más frecuentes en hombres mientras que las de gato lo son en mujeres (2:1), y que dos tercios de las víctimas de mordeduras de perro son niños y adolescentes, siendo $25 \%$ de las víctimas de edad menor a 6 años, mientras que dos tercios de las víctimas de mordeduras de gato tienen entre 20 y 35 años ${ }^{4,9}$.

En cuanto a la localización de la mordedura, 70 y $80 \%$ de las mordeduras producidas por ambas especies se localizan en manos, brazos y piernas y entre 10 y $30 \%$

\begin{tabular}{|c|c|c|}
\hline Fuente & Aeróbica & Anaeróbica \\
\hline \multirow[t]{8}{*}{ Perro } & Pasteurella spp. & Fusobacterium spp. \\
\hline & Streptococcus spp. & Porphyromonas spp. \\
\hline & Staphylococcus spp. & Prevotella spp. \\
\hline & Neisseria spp. & Propionibacterium spp. \\
\hline & Corynebacterium spp. & Bacteroides spp. \\
\hline & Moraxella spp. & Peptostreptococcus spp. \\
\hline & Enterococcus spp. & \\
\hline & Capnocytophaga canimorsus & \\
\hline \multirow[t]{8}{*}{ Gato } & Pasteurella spp. & Fusobacterium spp. \\
\hline & Streptococcus spp. & Porphyromonas spp. \\
\hline & Staphylococcus spp. & Bacteroides spp. \\
\hline & Neisseria spp. & Prevotella spp. \\
\hline & Moraxella spp. & Propionibacterium spp. \\
\hline & Corynebacterium spp. & \\
\hline & Enterococcus spp. & \\
\hline & Bartonella henselae & \\
\hline
\end{tabular}

se localizan en la cabeza, nuca y parte anterior del cuello. Hasta $90 \%$ de las heridas en niños bajo 5 años de edad se localizan en la cara y la parte anterior del cuello y éstas son producidas fundamentalmente por perros ${ }^{9}$.

El interés de la mordedura no radica únicamente en la destrucción de los tejidos donde se produce, sino también, en la posibilidad de la penetración de microorganismos de la boca del animal que pueden causar infección del individuo que ha sido mordido. El riesgo de infección puede aumentar según los siguientes factores:

Naturaleza de la herida: las mordeduras punzantes ( $1 \mathrm{~mm}$ de diámetro), las heridas que producen destrucción de tejidos marcada, con edema y con poca perfusión, en cara, pies, manos, genitales, articulaciones y prótesis articulares ${ }^{9}$. Las mordeduras en manos y muñecas han sido consideradas como un riesgo mayor de infección ${ }^{10}$.

Especie agresora: entre 30 y $50 \%$ de las mordeduras de gato se infectan frente al 5-25\% de las mordeduras de perro $^{4,9,11-13}$.

Características del paciente: niños bajo dos años de edad, pacientes con las siguientes comorbilidades: inmunodeprimidos, enfermedad hepática crónica, alcoholismo, asplenia, cáncer, diabetes mellitus y en tratamiento con corticosteroides, estasis venosa o linfática en el área de la herida y portadores de prótesis 9 .

La mayoría de las infecciones por mordeduras de perros y gatos presentan una etiología bacteriana mixta hallándose una mezcla de microorganismos aerobios y anaerobios que proceden, tanto de la boca del agresor como, más raramente, de la piel del agredido ${ }^{4,7,14}$. La Tabla 1 recoge las bacterias más comúnmente aisladas en la microbiota oral del perro y el gato, ordenadas según la frecuencia de aislamiento.

Entre las bacterias más frecuentemente aisladas, Staphylococcus spp se identifica en $50 \%$ de las mordeduras producidas por perros y gatos ${ }^{4,9}$. Pasteurella $\mathrm{spp}$ se aísla en $50 \%$ de los cultivos de mordeduras de perro y $75 \%$ de las ocasionadas por gato g, $^{7,12,15}$. Por otra parte, Capnocytophaga canimorsus forma parte de la microbiota comensal de perros (entre 22 y $74 \%$ de los perros sanos) y gatos (en torno a $17 \%$ de los animales sanos) ${ }^{16,17}$.

Una magnitud de la potencial gravedad de estas infecciones se refleja en el desenlace fatal de $60 \%$ de los casos de sepsis por C. canimorsus mientras que en la forma de localización meníngea se describe una letalidad de $5 \%{ }^{18}$.

En base a lo anteriormente descrito, y teniendo en cuenta el incremento en el número de animales de compañía que ha tenido lugar en los últimos años, el objetivo de esta revisión es conocer las principales bacterias implicadas en las infecciones provocadas por mordedura de perro y gato en humanos, así como los factores predisponentes (o comorbilidades) que favorecen la aparición de la infección y su diseminación.

Para ello, se formuló la siguiente pregunta PICO 
se muestra a continuación y se especifica en el diagrama de flujo siguiendo el criterio PRISMA ${ }^{20}$ (Figura 1). Se utilizaron los siguientes criterios de búsqueda: (("Bites, Human"[Mesh]) OR "Bites and Stings"[Mesh]) AND "Infection"[Mesh]. Se incluyeron casos clínicos, series de casos o estudios retrospectivos en los que se estudiaba la naturaleza de una infección provocada por mordedura de perro o gato en humanos y en los que se identificó el agente causal de la infección. Para su inclusión sólo se tuvieron en cuenta artículos escritos en inglés o español y publicados entre los años 2000 y 2019.
La búsqueda inicial arrojó un total de 311 trabajos

Se realizó una búsqueda bibliográfica en la base de datos PubMed, llevada a cabo entre el 1 de agosto y el 21 de octubre de 2019. La estrategia completa de búsqueda os principales agentes microbianos aislados que cursan con infecciones locales o sistémicas (outcome) en humanos (patients)?

\section{Materiales y Métodos}

PubMed (("Bites, Human"[Mesh]) OR "Bites and Stings"[Mesh]) AN D "Infection"[Mesh] $N=311$

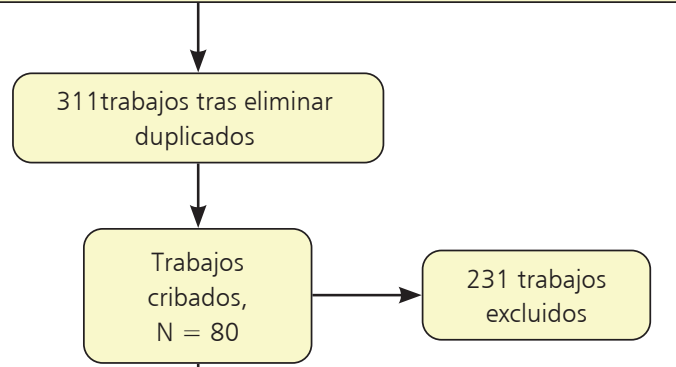

Figura 1. Diagrama de flujo de los estudios incluidos. 
tras eliminar aquellos que se encontraban duplicados. Un total de 231 trabajos fueron eliminados por título y resumen. Posteriormente se realizó un nuevo cribado con el que se obtuvo un total de 57 trabajos que se leyeron a texto completo. Un total de 32 trabajos a texto completo fueron excluidos por no especificar el agente causante de la infección (17 trabajos), porque la infección afectaba a otros animales (tres trabajos) o porque la infección se había producido por contacto (lametazos) sin mordedura (tres trabajos). El resto de los motivos de exclusión quedan detallados en la Figura 1.

Finalmente, el estudio incluyó 24 trabajos en la síntesis cualitativa que cumplían los criterios de inclusión establecidos.

\section{Resultados}

Se seleccionó un total de 24 artículos que se incluyeron en la revisión. La Tabla 2 muestra los datos recopilados de los estudios. 23 trabajos eran casos clínicos, una serie de seis $\operatorname{casos}^{21}$ y otra de tres casos ${ }^{13}$. La muestra total de los trabajos seleccionados fue de 31 pacientes, 17 varones y 14 mujeres, con una edad entre los 19 meses y los 68 años.

En el presente trabajo, únicamente se tuvieron en cuenta los casos de mordeduras provocadas por perro o gato y cuyas víctimas posteriormente desarrollaron infección. La principal especie mordedora fue el perro con una afectación de 20 individuos (17 casos clínicos individuales, una serie presentó tres casos) $)^{5,6,11,22-34}$. Por otra parte, 12 individuos fueron afectados por mordedura de gato que cursó con infección (de éstos, hubo seis publicados como casos clínicos y otros seis publicados en dos series de casos, presentando tres casos cada uno) ${ }^{13,14,17,21,35-38}$.

En un total de 16 trabajos se describieron mordeduras en las manos $5,6,11,13-15,17,21,22,25,27,31,34-36,38$ de las cuales cuatro de ellas habían sido producidas por gatos ${ }^{13,21,36,38}$, mientras que tres trabajos describieron mordeduras en los brazos $^{21,30,32}$. Las mordeduras en la cara producidas en su totalidad por perros ${ }^{24,26,29}$ mientras que las mordeduras descritas en extremidades inferiores fueron producidas en todos los casos por gatos ${ }^{13,17,35,37}$.

El tiempo transcurrido entre la mordedura y la solicitud de asistencia hospitalaria osciló entre las pocas horas ${ }^{32}$ tras la mordedura del animal y las seis semanas ${ }^{21}$.

Los factores predisponentes o comorbilidades más frecuentes en los pacientes que desarrollaron la infección fueron la esplenectomía previa ${ }^{25,30,33,37}$, hepatitis B o C o alcoholismo $^{11,34}$ y las alteraciones del sistema inmune 14,23,36,38. En 18 casos los pacientes no presentaron factores predisponentes y, aun así, en cuatro de estos casos se produjo shock séptico ${ }^{5,15,27,31}$. En dos casos en los que la mordedura se produjo en la rodilla, los pacientes presen- taban artroplastia o prótesis de rodilla que favorecieron la progresión de la infección ${ }^{17,39}$.

Para esta revisión únicamente se tuvieron en cuenta aquellas publicaciones que incluían un aislamiento e identificación del patógeno causante de la infección utilizando para ello diversas técnicas de laboratorio. La bacteria más comúnmente aislada en mordedura de perro (19 pacientes) fue $C$. canimorsus $(57,9 \% 11 / 19)^{5,6,11,22,23,25}$, 27,30,31,33,34; seguida por especies de Staphylococcus $(10,5 \%$ $2 / 19)^{21,24}$ y Pasteurella $(15,8 \% 3 / 19)^{15,21}$. Por otra parte, en las mordeduras de gato (12 pacientes) la bacteria más frecuentemente aislada fue Pasteurella multocida $(58,3 \%$ $7 / 12)^{17,35,36}$, aunque también se halló un caso provocado por Capnocytophaga canis $(8,3 \% 1 / 12)$ y otro por Bacteroides pyogenes $(8,3 \% 1 / 12)^{14,38}$.

Capnocitophaga canimorsus produjo infección generalizada grave (sepsis) en 12 pacientes $(38,7 \% 12 / 31)^{5,6,11,22,2}$ $3,25,27,30,31,33,34,37$. Esta bacteria fue trasmitida por mordedura de gato sólo en un individuo $(8,3 \% 1 / 12)^{37}$.

En 17 trabajos, se prescribió profilaxis antimicrobiana. Los fármacos prescritos con mayor frecuencia, tanto para la profilaxis post-mordedura como para el tratamiento post-identificación del microorganismo causante, fueron $\beta$-lactámicos con inhibidores de $\beta$-lactamasas (amoxicilina/ácido clavulánico, ampicilina/sulbactam y piperacilina/ tazobactam), cefalosporinas, metronidazol y clindamicina, tanto por vía oral como por vía intravenosa.

Dos pacientes que presentaban mordedura de perro fallecieron, una mujer y un hombre en la cuarta década de la vida y con factores predisponentes. En ambos casos, la mordedura se produjo en la mano y la infección había sido causada por $C$. canimorsus; en los dos se había prescrito profilaxis antimicrobiana inmediatamente tras la mordedura ${ }^{25,34}$.

\section{Discusión}

El incremento poblacional de las mascotas ha hecho que la importancia de las mordeduras en la clínica de urgencias cobre una mayor importancia en los últimos años. Según recientes estudios, C. canimorsus es la bacteria más frecuentemente aislada en los casos de mordedura de perro que cursan con infección, siendo la prevalencia de infección por esta bacteria de 0,5 a 4,1 casos por millón ${ }^{11}$. En el presente estudio, esta bacteria fue aislada en 57,9\% (11/19) de las infecciones causadas por mordedura de perro.

El género Capnocytophaga se encuentra en 22 a $74 \%$ de los perros sanos y tiene 9 especies ${ }^{5}$; sin embargo, en los estudios consultados solamente se encontraron publicaciones sobre la especie C. canimorsus ${ }^{5,6,11,22,23,25,27,30,31,33,34}$ y una única publicación sobre infección causada por $C$. canis provocada, además, por mordedura de gato $(8,3 \%$ $1 / 12)^{40}$. 


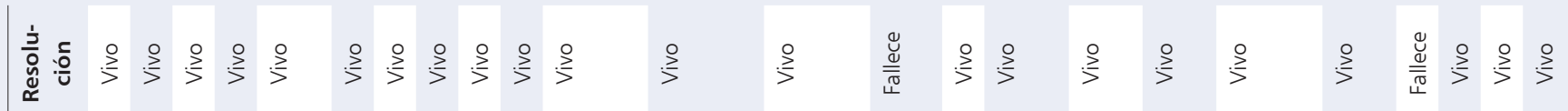

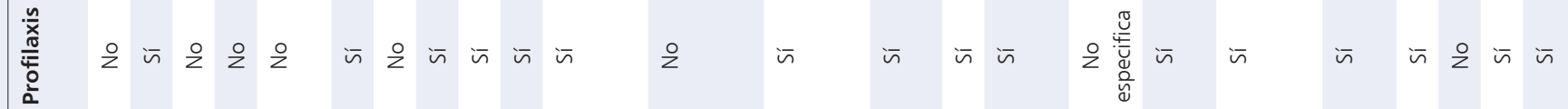

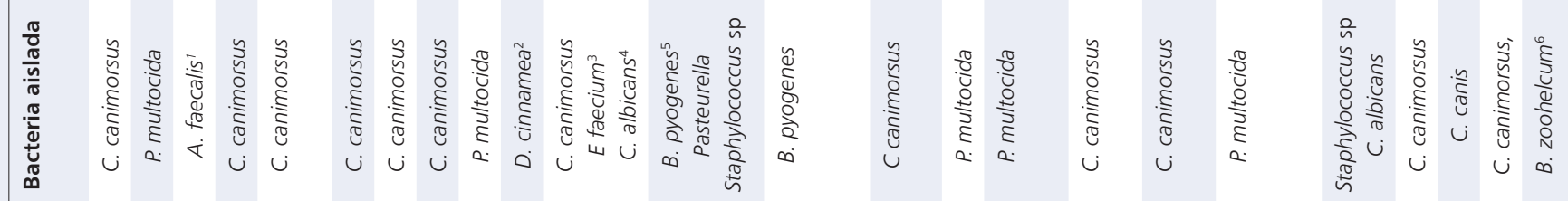

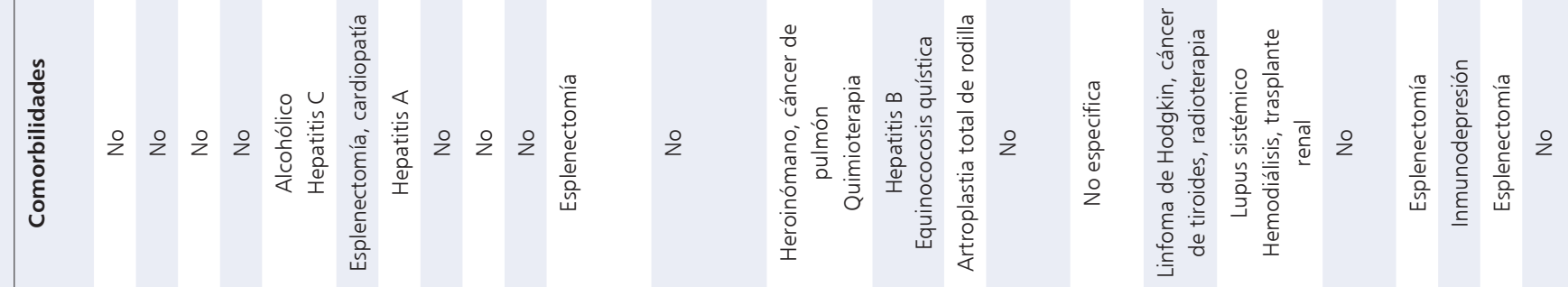

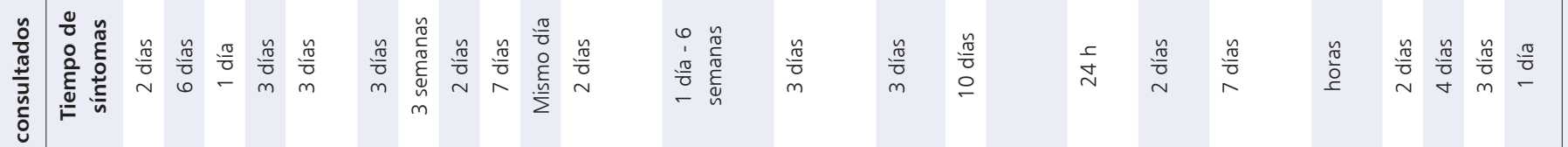

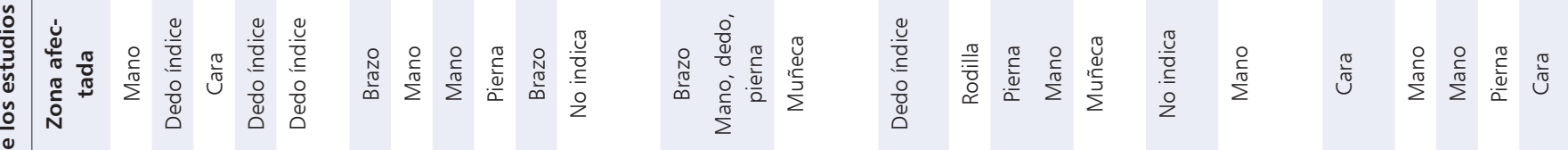

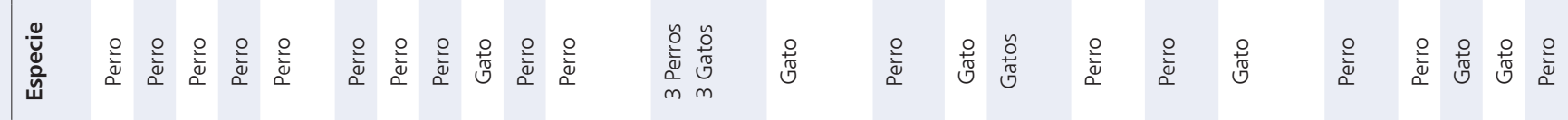

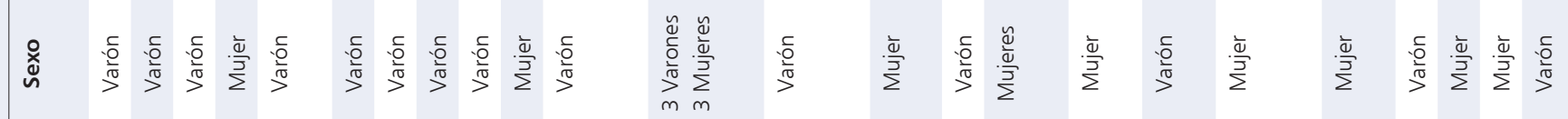

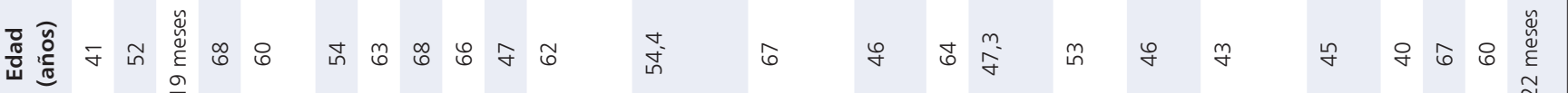


Tal y como se observa en nuestro estudio, durante los últimos 20 años existe una presencia moderada de casos de mordedura con infección provocada por C. canimorsus. Autores han considerado un incremento en el número de casos con respecto a años anteriores. Probablemente este hecho, no se relaciona con el incremento en la incidencia de esta bacteria, sino con la dificultad que ha tenido durante años su identificación. El éxito del diagnóstico de las bacterias en el laboratorio depende de la relación laboral establecida entre microbiólogos y médicos tratantes, que debe ser estrecha y positiva y con una comunicación

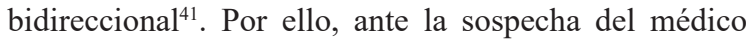
clínico de infección por bacterias de difícil aislamiento, tal y como es $C$. canimorsus, se debe de comunicar este hecho de inmediato al laboratorio de microbiología para garantizar el éxito de su aislamiento e identificación. De igual forma, el equipo de microbiología debe orientar al médico clínico sobre la selección, recolección y transporte de las muestras, ya que muchas de estas bacterias pueden verse comprometidas si el manejo no es adecuado, dando lugar a resultados engañosos. Este hecho perjudicaría directamente las medidas terapéuticas $\mathrm{y}$, eventualmente, la salud del paciente ${ }^{41}$. En esta línea se ha definido que, mientras en las mordeduras con sospecha de infección por Pasteurella spp. se deben tomar hemocultivos, en el caso de $C$. canimorsus y Actinobacillus spp. se deben tomar muestras de tejido dañado (mediante biopsia o aspiración) para posteriormente realizar un cultivo aérobico o anaeróbico. Tal y como se observa en estos dos casos, el conocimiento de las diferentes muestras que deben de recogerse en función del agente microbiano implicado es fundamental para incrementar la fiabilidad del diagnóstico. Según estos autores, el éxito en el diagnóstico de enfermedades infecciosas se logra aplicando un conocimiento profundo de la ciencia médica y de laboratorio junto con los principios de epidemiología y farmacocinética de los antimicrobianos e integrando una visión estratégica de las interacciones hospedero-parásito ${ }^{41}$.

Como se ha mencionado, C. canimorsus es una bacteria difícil de aislar, con un crecimiento muy lento y que puede tardar hasta 14 días en crecer en un medio de cultivo. En los últimos años diferentes técnicas laboratoriales han sido realizadas encaminadas a la detección de este patógeno. Distintos autores han considerado la identificación del patógeno mediante el uso de sondas de ADN (ARNr 16S RPC). Por otra parte, la espectrometría de masas por tiempo de vuelo de ionización por desorción láser asistida por matriz (MALDI-TOF MS) constituye una valiosa herramienta en la identificación de este patógeno de difícil cultivo. El estudio realizado por Magnette y cols. ${ }^{42}$ proporcionó una identificación confiable a nivel de especie para $100 \%$ de los casos estudiados con este patógeno. En ambos casos, se trata de técnicas relativamente modernas, por lo que es probable que este sea el motivo del incremento en la detección del patógeno $0^{5,11,27}$.

Se ha descrito una tasa de mortalidad asociada a shock séptico por $C$. canimorsus entre 30 y $60 \% 0^{5,11,27}$. En esta serie, dos individuos de los 12 que presentaron infección por esta bacteria, murieron ${ }^{25,34}$. El riesgo de sepsis y la muerte por infección por esta bacteria se asocia con la presencia de comorbilidades (tales como asplenia, pacientes inmunocomprometidos, patología hepática y alcoholismo $)^{6,11,23,25,30,33,34}$. En nuestro estudio, los dos casos que concluyeron con la muerte del paciente presentaban factores predisponentes (hepatitis B con equinococosis quística descubierta post-mortem, y esplenectomía, respectivamente). Por otra parte, es importante destacar que, en en esta revisión, se hallaron casos de infección grave en pacientes sin factores predisponentes previos s, $27,31,43,44^{\text {. }}$

El género Pasteurella spp. es el anaerobio facultativo más comúnmente aislado en las mordeduras de gato, siendo $P$. multocida la especie más común $(58,3 \%$ $7 / 12)^{13,17,35,36}$. En dos de estos casos los pacientes presentaban factores predisponentes ${ }^{17,36}$. Esta bacteria también fue aislada en un individuo que presentó mordedura de perro $(1 / 19)^{15}$. Otros agentes bacterianos también fueron identificados en las infecciones provocadas por mordeduras de perros y gatos; en un estudio se halló un caso de un paciente con infección causada por Alcaligenes faecalis tras mordedura de perro (1/19). Esta especie se halla en la microbiota del tracto digestivo en humanos no siendo patógeno por naturaleza; sin embargo, puede provocar infecciones oportunistas. Parece probable, por tanto, que en este caso procediese de una sobreinfección y su origen no se halle en la mordedura del perro en sí ${ }^{29}$. Se halló otro caso individual provocado por Bergeyella zoohelcum un bacilo gramnegativo aerobio que se halla en la microbiota oral normal de perros y gatos $(1 / 19)^{26}$. Finalmente, otro individuo presentó una infección provocada por Dietzia cinnamea tras una mordedura de perro (1/19). Esta bacteria, bacilo grampositivo cocoide, se halla en la placa dental de los perros y puede actuar como un patógeno oportunista ${ }^{32}$.

Los pacientes de los estudios seleccionados en esta revisión acudieron al servicio hospitalario mostrado sintomatología general como alteración del estado mental, dolor abdominal, náuseas, mialgia, diarrea, vómitos y distermia $^{27,34,36,37}$. En la exploración general, los casos clínicos incluidos coinciden en la presencia de signos tales como fiebre, hipotensión arterial, taquicardia y taquipnea ${ }^{17,24,34,37}$. Así mismo, dichas casuísticas describieron como complicaciones sistémicas más frecuentes la coagulación vascular diseminada (CID), fallo multiorgánico, meningitis y fallo renal ${ }^{11,27,33}$. Es importante, tener en cuenta que, aunque autores previos han considerado que el riesgo de desarrollo de infecciones depende de la presencia de factores predisponentes, el presente estudio 
mostró que en 10 publicaciones (12 pacientes) los pacientes no presentaron factores predisponentes y aun así en cuatro de estos casos se produjo shock séptico ${ }^{13,15,27,31}$.

Se ha descrito que las mordeduras de la mano tienen mayor riesgo de desarrollar la infección frente a las que se producen en otras localizaciones ${ }^{10}$. Esto queda justificado por la distribución anatómica de la mano. Los compartimentos cerrados y los planos fasciales en la mano junto con los nervios, huesos y articulaciones adyacentes a la superficie, hacen que la mano sea propensa a desarrollar infecciones del espacio profundo y osteomielitis ${ }^{17}$. Este hecho justifica la alta importancia de la inclusión de profilaxis antimicrobiana en el caso de individuos que hayan sido mordidos en la mano ${ }^{17}$.

Aunque la antibioprofilaxis ha sido considerada efectiva sólo en aquellos casos de mordeduras en la mano $^{17}$, la mayor parte de los trabajos establecen que las heridas punzantes y profundas, y también los pacientes que presentan comorbilidades, se benefician de esta profilaxis ${ }^{14-16,31,34,36}$. Por este motivo, se recomienda el uso de antimicrobianos de amplio espectro durante 3 a 5 días tras la mordedura ${ }^{5,6,11,27,38}$; se considera que la profilaxis antimicrobiana reduce en 2 a $28 \%$ el riesgo de infección. Dicha profilaxis debe complementarse con protección adecuada contra el tétanos y la rabia, así como el desbridamiento local de la herida ${ }^{28}$. En la presente revisión, en aquellos casos que cursaron con infección, inicialmente se pautó un tratamiento antimicrobiano empírico de amplio espectro que podía ser modificado o no, una vez que se aislaba la bacteria causante de la infección ${ }^{24,31,37}$. Teniendo en cuenta que la mayor parte de las bacterias causantes de este tipo de infecciones son susceptibles a $\beta$-lactámicos, se destacó el uso de amoxicilina/ácido clavulánico y ampicilina/ sulbactam, aunque también se indicó aminoglucósidos (gentamicina) y nitroimidazoles (metronidazol) 24,31,37,45. Estos hallazgos están de acuerdo con los resultados de nuestro trabajo, donde el antimicrobiano de elección más utilizado de forma empírica fue amoxicilina/ácido clavulánico.

\section{Conclusiones}

La infección sistémica producida por mordedura de perro o gato en el hombre se produce generalmente en pacientes con factores predisponentes o concomitantes; sin embargo, no se deben subestimar las lesiones de este tipo incluso en pacientes sanos. Entre las especies bacterianas presentes en las mordeduras destaca C. canimorsus en perros y $P$. multocida en gatos. Es recomendable prestar atención a este tipo de lesiones, así como recibir asistencia médica tras una mordedura y recibir una pauta de profilaxis antimicrobiana si la mordedura se produce en la mano, es profunda o existen comorbilidades. Por otro lado, es importante estar atento a la presencia de síntomas sistémicos que pueden llevar a la aparición de shock séptico con riesgo de muerte. Se debe notificar en los servicios hospitalarios el antecedente de mordedura por animal, ya que el conocimiento por parte del médico facilitará la toma de decisiones.

\section{Referencias bibliográfícas}

1.- (AMVAC) Asociación Madrileña de Veterinarios de Animales de Compañía A. Informe Sectorial 2010. Madrid; 2010. p. 28.

2.- Dowd K, Taylor M, Toribio J-A L M L, Hooker C, Dhand N K. Zoonotic disease risk perceptions and infection control practices of Australian veterinarians: call for change in work culture. Prev Vet Med. 2013; 111(1-2): 17-24. doi: 0.1016/j.prevetmed.2013.04.002.

3.- Epp T, Waldner C. Occupational health hazards in veterinary medicine: Zoonoses and other biological hazards. Can Vet J. 2012; 53(2): 14450. PMID: 22851775.

4.- Jaindl M, Oberleitner G, Endler G, Thallinger C, Kovar F M. Management of bite wounds in children and adults-an analysis of over 5000 cases at a level I trauma centre. Wien Klin Wochenschr. 2016; 128(9-10): 367-75. doi: 10.1007/s00508-015-0900-x.

5.- Ahmad S, Yousaf A, Inayat F, Anjum S. Capnocytophaga canimorsus-associated sepsis presenting as acute abdomen: do we need to think outside the box? Br Med J Case Rep. 2019; 12(3): e228167. doi: 10.1136/bcr-2018228167.

6.- Evans T J, Lyons OT, Brown A, Price N, Bell R E, Sallam M. Mycotic aneurysm following a dog bite: the value of the clinical history and molecular diagnostics. Ann Vasc Surg. 2016; 32: 130.e5--7. doi: 10.1016/j. avsg.2015.10.026.

7.- Kennedy S A, Stoll L E, Lauder A S. Human and other mammalian bite injuries of the hand: evaluation and management. J Am Acad Orthop Surg. e2015; 23(1): 47-57. doi: 10.5435/ JAAOS-23-01-47.

8.- Reisner I R. Differential diagnosis and management of human-directed aggression in dogs. Vet Clin North Am Small Anim Pract. 2003; 33(2): 303-20. doi: 10.1016/S01955616(02)00132-8.

9.- Rothe K, Tsokos M, Handrick W. Animal and human bite wounds. Dtsch Arztebl Int. 2015; 112(25): 433-43. doi: 10.3238/ arztebl.2015.0433.

10.- Medeiros IM, Saconato H. Antibiotic prophylaxis for mammalian bites (Review) Cochrane Collab. 2010;(2): 1-20. doi: 10.1002/14651858.CD001738.

11.- Elliott C, Byars J-P, Weinhardt B, Khalsa K. A dog's dinner: an interesting case presenting as gastroenteritis. Br Med J Case Rep. 2018; 11(1):e226131. doi: 10.1136/bcr2018-226131.

12.- Murray G E. Examining evidence on dog bite injuries and their management in children. Nurs Child Young People. 2017; 29(3): 35-9. doi: 10.7748/ncyp.2017.e859.

13.- Nocera N F, Desai K K, Granick M S. Cat bite cellulitis. Eplasty. 2014 Aug 8;14:ic25. PMID: 25210576.

14.- Madsen I R, Justesen U S. Bacteremia with Bacteroides pyogenes after a cat bite. J Clin Microbiol. 2011; 49(8): 3092-3. doi: 10.1128/ JCM.00250-11.

15.- Borges L, Oliveira N, Cassio I, Costa H. Sepsis-induced purpura fulminans caused by Pasteurella multocida. Case Reports. 2014; 19(1):bcr2013202441. doi: 10.1136/bcr-2013202441. 
16.- Ellis R, Ellis C. Dog and cat bites. Am Fam Physician. 2014; 90(4): 239-43. PMID: 25250997.

17.- Miranda I, Angulo M, Amaya J V. Infección aguda de prótesis total de rodilla tras mordedura y arañazo de gato: caso clínico y revisión de la bibliografía. Rev Esp Cir Ortop Traumatol. 2013; 57(4): 300-5. doi: 10.1016/j. recot.2013.04.006.

18.- Zajkowska J, Krol M, Falkowski D, Syed N, Kamienska A. Capnocytophaga canimorsus an underestimated danger after dog or cat bite - review of literature. Przegl Epidemiol. 2016; 70(2): 289-95. PMID: 27837588.

19.- Abrahamian F M, Goldstein E J C. Microbiology of animal bite wound infections. Clin Microbiol Rev. 2011; 24(2): 231-46. doi:10.1128/CMR.00041-10.

20.- Moher D, Liberati A, Tetzlaff J, Altman D G. Preferred reporting items for systematic reviews and meta-analyses: the PRISMA statement. Ann Intern Med. 2009; 151(4): 2649. doi: 10.1371/ journal.pmed.1000097.

21.- Lau J S Y, Korman T M, Yeung A, Streitberg R, Francis M J, Graham M. Bacteroides pyogenes causing serious human wound infection from animal bites. Anaerobe. 2016; 42: 172-5. doi: 10.1016/j.anaerobe.2016.10.008.

22.- O'Rourke G A, Rothwell R. Capnocytophaga canimorsus a cause of septicaemia following a dog bite: A case review. Aust Crit Care. 2011; 24(2): 93-9. doi: 10.1016/j.aucc.2010.12.002.

23.- Sacks R, Kerr K. A 42-year-old woman with septic shock: an unexpected source. J Emerg Med. 2012; 42 (3): 275-8. doi: 10.1016/j. jemermed.2008.09.020.

24.- Spagnoli A M, Monacelli G, Rizzo M I, Fallico $\mathrm{N}$, Scuderi N. Extensive facial trauma caused by dog bites in woman suffering from systemic fluconazole-resistant Candida infection. G Chir. 2011; 32(8-9): 376-8. PMID: 22018261.

25.- Stiegler D, Gilbert J D, Warner M S, Byard R W. Fatal dog bite in the absence of significant trauma: Capnocytophaga canimorsus infection and unexpected death. Am J Forensic Med Pathol. 2010; 31(2): 198-9. doi: 10.1097/ PAF.0b013e3181dfc98b.

26.- Yi J, Humphries R, Doerr L, Jerris R C, Westblade LF. Bergeyella zoohelcum associated with abscess and cellulitis after a dog bite. Pediatr Infect Dis J. 2016; 35(2): 214-6. doi: 10.1097/INF.0000000000000971.

27.- Dedy N J, Coghill S, Chandrashekar N K S, Bindra R R. Capnocytophaga canimorsus sepsis following a minor dog bite to the finger: case report. J Hand Surg Am. 2016; 41(1): 814. doi: 10.1016/j.jhsa.2015.10.011.

28.- Ambro B T, Wright R J, Heffelfinger R N Management of bite wounds in the head and neck. Facial Plast Surg. 2010; 26(6): 456-63. doi: $10.1055 / \mathrm{s}-0030-1267720$.

29.- Chu A S, Harkness J. Alcaligenes faecalis cellulitis after a dog bite: case report and literature review. Pediatr Emerg Care. 2017; 33(7): 497-8. doi: 10.1097/ PEC.0000000000000645.

30.- Ensor C R, Russell S D, Wittstein I S, Conte J V. Asplenic heart transplant candidate with a left ventricular assist system. Prog Transplant. 2011;21(2):121-3. doi: 10.7182/ prtr.21.2.1358461374r76423.

31.- Hawkins J, Wilson A, McWilliams E. «Biting the hand that feeds»: Fever and altered sensorium following a dog bite. Emerg Med J. 2011; 28(12): 1071-3. doi: 10.1136/ emj.08.2010.3265rep.

32.- Hirvonen J J, Lepistö I, Mero S, Kaukoranta S-S. First isolation of Dietzia cinnamea from a dog bite wound in an adult patient. J Clin Microbiol. 2012; 50(12): 4163-5. doi: 10.1128/ JCM.01939-12.

33.- Hloch O, Mokra D, Masopust J, Hasa J, Charvat J. Antibiotic treatment following a dog bite in an immunocompromized patient in order to prevent Capnocytophaga canimorsus infection: a case report. BMC Res Notes. 2014;7(1):432. doi: 10.1186/1756-0500-7-432.

34.- Matulionytė R, Lisauskienė I, Kèkštas G, Ambrozaitis A. Two dog-related infections leading to death: overwhelming Capnocytophaga canimorsus sepsis in a patient with cystic echinococcosis. Medicina (Kaunas). 2012; 48(2): 112-5. doi: 10.3390/ medicina48020011.

35.- Heydemann J, Heydemann J S, Antony S. Acute infection of a total knee arthroplasty caused by Pasteurella multocida: a case report and a comprehensive review of the literature in the last 10 years. Int J Infect Dis. 2010; 14:e242--e245. doi: 10.1016/J. IJID.2009.09.007.

36.- Schneider J R, White G W, Dejesus E F. Pasteurella multocida-infected expanded polytetrafluoroethylene hemodialysis access graft. Ann Vasc Surg. 2012; 26(8): 1128.e151128.e17. doi: 10.1016/j.avsg.2012.03.007.

37.- Teo K G, Anavekar N S, Yazdabadi A, Ricketts S. Asplenic fulminant sepsis secondary to a dog bite complicated by toxic epidermal necrolysis/Stevens-Johnson syndrome. J New
Zeal Med Assoc. 2012; 125(1358): 74-7. PMID: 22864159.

38.- Taki M, Shimojima Y, Nogami A, Yoshida T, Suzuki M, Imaoka K, et al. Sepsis caused by newly identified Capnocytophaga canis following cat bites: $C$. canis is the third candidate along with C. canimorsus and $C$. cynodegmi causing zoonotic infection. Intern Med. 2018; 57(2): 273-7. doi: 10.2169/ internalmedicine.9196-17.

39.- Vélez F A, Laboy Ortíz I E, López R, Sánchez A, Colón M, Martínez J H. Pasteurella multocida: a nightmare for a replaced joint and the challenge to save it. Bol Asoc Med Puerto Rico. 2014; 106(1): 43-5. PMID: 24791364

40.- Suzuki M, Imaoka K, Haga Y, Mohri M, Nogami A, Shimojima Y, et al. Characterization of three strains of Capnocytophaga canis isolated from patients with sepsis. Microbiol Immunol. 2018; 62(9): 567-73. doi: 10.1111/1348-0421.12642.

41.- Baron E J, Miller J M, Weinstein M P, Richter S S, Gilligan P H, Thomson R B, et al. A guide to utilization of the microbiology laboratory for diagnosis of infectious diseases: 2013 Recommendations by The Infectious Diseases Society of America (IDSA) and the American Society for Microbiology (ASM). Clin Infect Dis. 2013; 57(4). e22-e121. doi: 10.1093/cid/ cit278.

42.- Magnette A, Huang T D, Renzi F, Bogaerts P, Cornelis G R, Glupczynski Y. Improvement of identification of Capnocytophaga canimorsus by matrix-assisted laser desorption ionization-time of flight mass spectrometry using enriched database. Diagn Microbiol Infect Dis. 2016; 84(1): 12-5. doi: 10.1016/j. diagmicrobio.2015.09.016

43.- Nishioka H, Kozuki T, Kamei H. Capnocytophaga canimorsus bacteremia presenting with acute cholecystitis after a dog bite. J Infect Chemother. 2015; 21(3): 215-7. doi: 10.1016/j.jiac.2014.09.001.

44.- O'Shaughnessy S M, Broderick L, Walsh J, Schaffer K, Westbrook A. Canis caveat (Beware of the Dog) - septic shock due to Capnocytophaga canimorsus contracted from a dog bite. Ir Med J. 2018; 111(10): 842. PMID: 30560637.

45.- Hindler J, Richter S, Bernard M, Bodeys-Jones S, Castanheira M, Citron D, et al. Methods for antimicrobial dilution and disk susceptibility testing of infrequently isolated or fastidious bacteria. Clinical and Laboratory Standards Institute. 3rd ed. 2016. 